\title{
Celecoxib coupled to dextran via a glutamic acid linker yields a polymeric prodrug suitable for colonic delivery
}

This article was published in the following Dove Press journal:

Drug Design, Development and Therapy

30 July 2015

Number of times this article has been viewed

\author{
Yonghyun Lee ${ }^{1,2, *}$ \\ Jungyun $\mathrm{Kim}^{1, *}$ \\ Wooseong Kim ${ }^{1, *}$ \\ Joon $\mathrm{Nam}^{1, *}$ \\ Seongkeun Jeong' \\ Sunyoung Lee' \\ Jin-Wook Yoo' \\ Min-Soo Kim' \\ Yunjin Jung' \\ 'College of Pharmacy, Pusan National \\ University, Busan, ${ }^{2}$ Bio-Nanomedicine \\ Laboratory, Department of Biological \\ Sciences, Korea Advanced Institute \\ of Science and Technology, Daejeon, \\ Republic of Korea \\ *These authors contributed equally \\ to this work
}

Correspondence: Yunjin Jung College of Pharmacy, Pusan National University, 2, Busandaehak-ro 63beon-gil, Geumjeong-gu, Busan 609-735, Republic of Korea

$\mathrm{Tel}+825 \mid 5102527$

Fax +82 5I 5I36754

Email jungy@pusan.ac.kr
Abstract: Celecoxib, a selective cyclooxygenase-2 inhibitor, is potentially useful for the treatment of colonic diseases such as colorectal cancer and colitis. However, the cardiovascular toxicity of celecoxib limits its routine use in the clinic. Generally, colon-specific delivery of a drug both increases the therapeutic availability in the large intestine and decreases the systemic absorption of the drug, most likely resulting in enhanced therapeutic effects against colonic diseases such as colitis and reduced systemic side effects. To develop a colon-specific prodrug of celecoxib that could reduce its cardiovascular toxicity and improve its therapeutic activity, dextran-glutamic acid-celecoxib conjugate (glutam-1-yl celecoxib-dextran ester [G1CD]) was prepared and evaluated. While stable in $\mathrm{pH} 1.2$ and 6.8 buffer solutions and small-intestinal contents, G1CD efficiently released celecoxib in cecal contents. Oral administration of G1CD to rats delivered a larger amount of celecoxib to the large intestine than free celecoxib. G1CD prevented the systemic absorption of celecoxib and did not decrease the serum level of 6-ketoprostaglandin $\mathrm{F}_{1 \alpha}$, an inverse indicator of cardiovascular toxicity of celecoxib. Collectively, G1CD may be a polymeric colon-specific celecoxib prodrug with therapeutic and toxicological advantages.

Keywords: colon-specific drug delivery, dextran, celecoxib, prodrug, cardiovascular toxicity

\section{Introduction}

Nonsteroidal anti-inflammatory drugs have been studied as chemopreventive agents for colorectal cancer (CRC). ${ }^{1,2}$ Since chemoprevention involves the long-term use of oral agents, the numerous side effects of nonsteroidal anti-inflammatory drugs have led to the search for safer drugs. ${ }^{3}$ Selective cyclooxygenase (COX)-2 inhibitors including celecoxib have been developed as safer alternatives to conventional COX inhibitors. ${ }^{4}$ A number of in vitro and in vivo experiments have demonstrated the effectiveness of coxibs in the chemoprevention of CRC and for the treatment of familial adenomatous polyposis. ${ }^{5-7}$ In addition, selective COX-2 inhibitors have beneficial effects against experimental colitis. ${ }^{8-10}$ However, clinical studies have revealed that long-term use of coxibs increases the risk of serious dose-associated cardiovascular events. ${ }^{1-14}$ Considering that chemoprevention or treatment of colonic diseases such as CRC and colitis requires long-term pharmacotherapy, celecoxib cannot be routinely recommended for this indication owing to its potential to cause cardiovascular events.

In general, colon-targeted delivery of an orally administered drug is a pharmaceutical strategy that is used to achieve efficient treatment of colonic diseases such as CRC and colitis, and reduce systemic side effects. These beneficial effects result from increased therapeutic concentration at the target site (large intestine) while restricting systemic absorption. ${ }^{15}$

A colon-specific prodrug needs to reach the colon after oral administration and activation in the gut lumen. Hydrophilic small molecules or polymers are used as 
colon-specific promoieties to limit the systemic absorption in the upper intestine. The linkage between a drug and the promoieties should be chemically and enzymatically stable during the transit through the stomach and small intestine. ${ }^{15}$ After delivery to the colon, the prodrug is presumed to be activated by enzymes originating from the microbes that are abundant in that portion of the alimentary canal. ${ }^{16}$

Dextran, a nonstarch polysaccharide with a linear $\alpha-1,6-$ glucopyranose chain and $\alpha$-1,3-glucopyranose branching, is not digestible by host glycosidases but is degraded readily by dextranases derived from microbes residing in the large intestine. ${ }^{15}$ For this reason, dextran has been used as a colon-specific polymeric carrier. There are many papers demonstrating that dextran-drug conjugates can deliver drugs specifically to the large intestine. ${ }^{15,17}$ During transit through the gastrointestinal tract, a dextran-drug conjugate does not release the drug in the upper intestine, presumably because the steric hindrance of the polymer matrix prevents enzymatic action at the linkage between dextran and the drug. Release of the drug takes place only after depolymerization of the dextran matrix by endodextranase, which is found in the colon as a result of the high bacteria count. ${ }^{18}$

Dextran-succinic acid-celecoxib was reported as a dextran-based polymeric prodrug of celecoxib. ${ }^{19}$ However, we found that succinyl celecoxib (SC) was not cleaved effectively enough to liberate celecoxib in the cecal contents, which may lead to inefficient release of celecoxib after colonic delivery. In this study, dextran-glutamic acid-celecoxib, where glutamic acid with a dicarboxylic acid was used as a linker, was prepared and evaluated as a polymeric colon-specific prodrug of celecoxib. Toxicological and therapeutic advantages were suggested by providing data on disposition and serum 6-ketoprostaglandin $\mathrm{F}_{1 \alpha}$ (6-keto$\mathrm{PGF}_{1 \alpha}$ ) after oral administration of the polymeric prodrug.

\section{Materials and methods \\ Materials}

1,1'-Carbonyldiimidazole (CDI), dextranase (Penicillium sp.), and 3,5-dinitrosalicylic acid (DNS) were purchased from Sigma Chemical Co. (St Louis, MO, USA). Succinic anhydride and 5-benzyl $N$-(Boc)-glutamate were purchased from Tokyo Chemical Industry (Tokyo, Japan). Dextran (molecular weight: 15-20 kDa) was purchased from Fluka (Sigma Chemical Co.). Celecoxib was ether-extracted from Celebrex capsules (Pfizer, Inc., New York, NY, USA). All other chemicals were reagent grade, commercially available products. Buffer solutions ( $\mathrm{pH} 1.2$ and 6.8) were prepared as described in USP XXIII. Thin layer chromatographys (TLCs) were performed on Merck Kieselgel 60 F254. The high-performance liquid chromatography (HPLC) system consisted of a Model 306 pump, a Model 117 UV detector, a Model 234 autoinjector, and a Model 805 manometric module from Gilson (Middleton, WI, USA). A symmetry column C18 (Waters, Milford, MA, USA) $(250 \times 4.6 \mathrm{~mm})$ with a guard column (Waters, $3.9 \times 20 \mathrm{~mm}$ ) was used. Six-week-old male Sprague Dawley rats (Samtako Bio Korea, Kyeong-gi-do, South Korea) were housed in the university animal facility with controlled temperature, humidity, and dark/light cycle. The animal protocol used in this study has been reviewed and approved by the Pusan National University-Institutional Animal Care and Use Committee (PNU-IACUC) on their ethical procedures and scientific care.

\section{HPLC analysis}

Standard solutions of celecoxib in various biological specimens were prepared as described previously. ${ }^{20}$ Standard or blank solution ( $1 \mathrm{~mL}$ ) was mixed on a vortex mixer for 5 minutes, centrifuged at $10,000 \times g$ for 10 minutes at $4^{\circ} \mathrm{C}$ and filtered through a membrane filter $(0.45 \mu \mathrm{m})$. The filtrate $(20 \mu \mathrm{L})$ was injected on a symmetry $\mathrm{C}_{18}$ column, which was eluted with the mobile phase at a flow rate of $1 \mathrm{~mL} / \mathrm{min}$. The mobile phase consisted of $60 \%$ acetonitrile (ACN) in 0.067 $\mathrm{M}$ phosphate buffer ( $\mathrm{pH} 4.0$ ) containing $0.1 \%$ trifluoroacetic acid, which was filtered through $0.45 \mu \mathrm{m}$ membrane filter before use. The eluate was monitored at $273 \mathrm{~nm}$ by a UV detector measuring the absorption with a sensitivity of absorbance units full scale (AUFS) 0.01. Gilson 712 software was used for data analysis. The retention time of celecoxib, glutam-1-yl celecoxib (G1C), and SC was 9.13, 3.20, and 9.33 minutes, respectively.

\section{Preparation of SC}

Succinic anhydride (2, $0.33 \mathrm{mg}, 3.30 \mathrm{mmol})$ was added to a solution of celecoxib (1, $0.50 \mathrm{~g}, 1.31 \mathrm{mmol})$ in ACN (15 mL) in the presence of triethylamine (TEA, $4.3 \mathrm{~mL}$ ). The reaction mixture was stirred at $55^{\circ} \mathrm{C}$ for 4 hours. The mixture was concentrated by evaporation and washed with $1 \mathrm{M}$ hydrochloric acid $(\mathrm{HCl})$ to afford the final product 1-succinylaminosulfonyl4-(5-[4-methylphenyl]-3-[trifluoromethyl] pyrazol-1-yl) benzene (3) as white powder. Synthetic scheme is shown in Figure 1A. Melting point (mp): $119^{\circ} \mathrm{C}-124^{\circ} \mathrm{C}$; infrared (IR) (Nujol, $\left.\mathrm{cm}^{-1}\right)$ : 1,718 (C=O SO $\left.2 \mathrm{NHCO}\right), 1,658(\mathrm{C}=\mathrm{O}$, carboxylic, shoulder); proton nuclear magnetic resonance ( $\left.{ }^{1} \mathrm{H}-\mathrm{NMR}\right)$ (dimethylsulfoxide [DMSO]- $\left.d_{6}, \mathrm{ppm}\right): 2.30(\mathrm{~s}, 3 \mathrm{H}), 2.35$ (t, $2 \mathrm{H}, J=7.5 \mathrm{~Hz}), 2.45$ (t, 2H, $J=6.5 \mathrm{~Hz}), 7.17(\mathrm{~s}, 1 \mathrm{H}), 7.16-7.21$ (m, 4H), 7.56 (d, 2H, J=8.5 Hz), 7.95 (d, 2H, J=8.5 Hz); Elemental analysis: $\mathrm{C}_{21} \mathrm{H}_{18} \mathrm{~F}_{3} \mathrm{~N}_{3} \mathrm{O}_{5} \mathrm{~S}$ (481.09) Calcd: C, 52.39; H, 3.77; N, 8.73; Found: C, 52.44; H, 3.82; N, 8.75. 
A

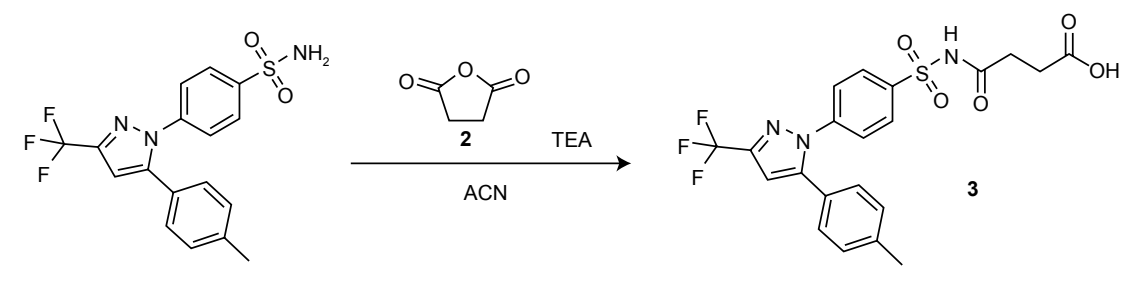

B

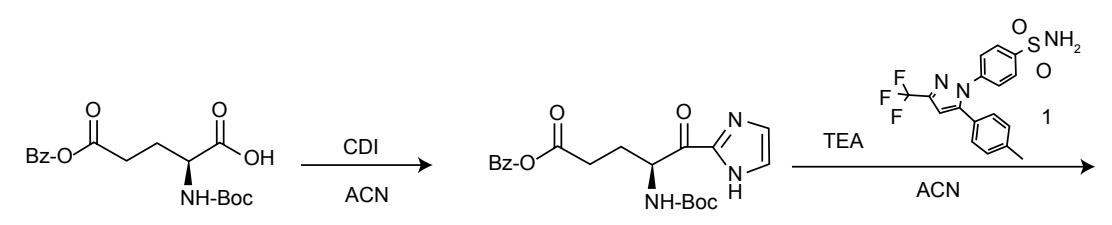<smiles>CCCCC(=O)OCC[C@H](NC(=O)c1ccccc1)C(=O)NS(=O)(=O)c1ccc(-n2nc(C(F)(F)F)cc2-c2ccc(C)cc2)cc1</smiles>

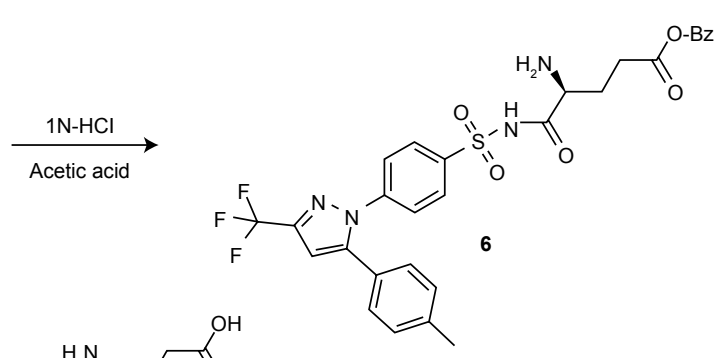

$\underset{\mathrm{H}_{2} \mathrm{O}}{\stackrel{1 \mathrm{~N}-\mathrm{NaOH}}{\longrightarrow}}$<smiles>Cc1ccc(-c2cc(C(F)(F)F)nn2-c2ccc(S(=O)(=O)NC(C)N)cc2)cc1</smiles>

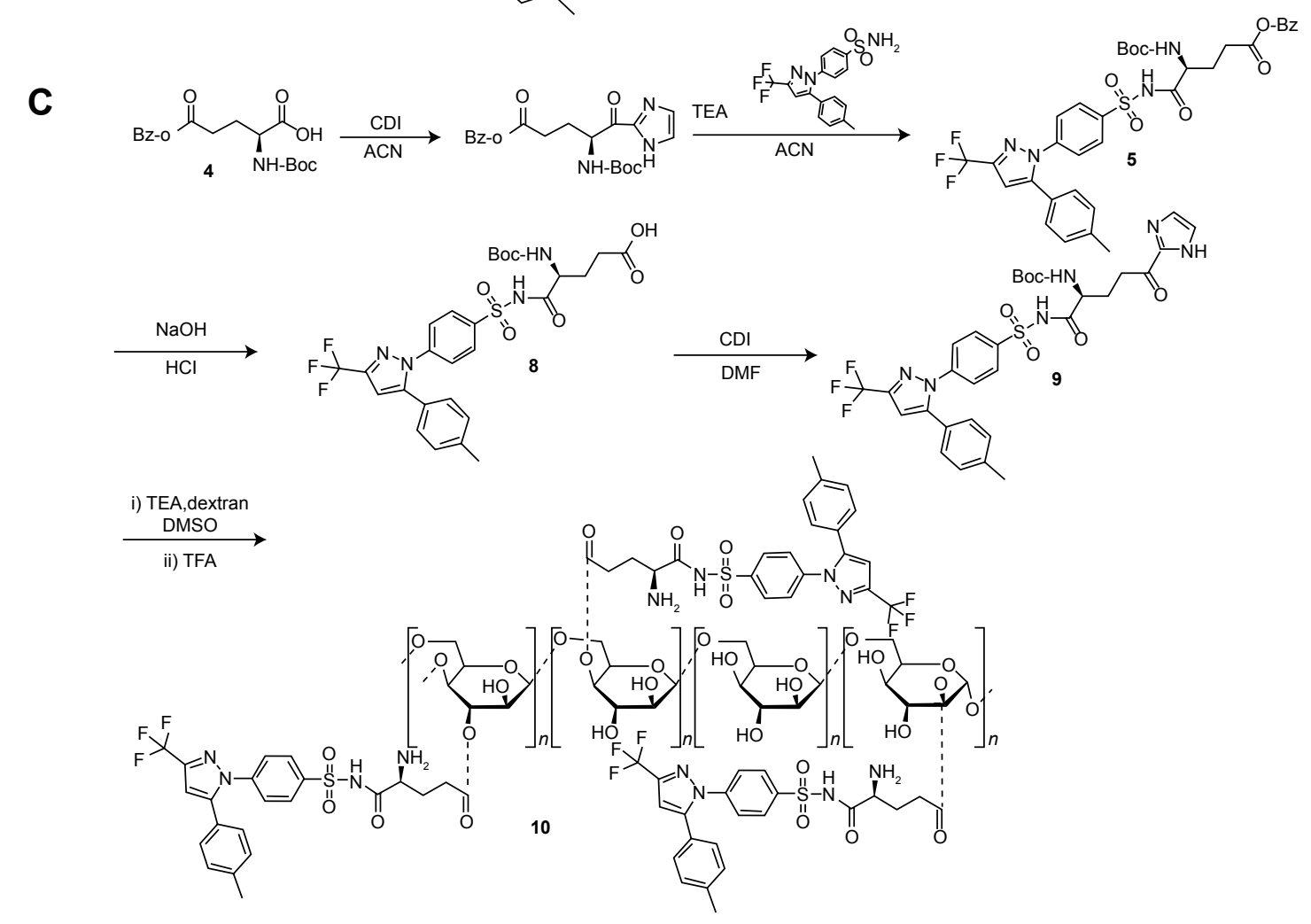

Figure I Synthetic scheme of celecoxib derivatives.

Notes: (A) Synthesis of succinyl celecoxib (3, SC); (B) synthesis of glutam-I-yl celecoxib (7, GIC); (C) synthesis of glutam-I-yl celecoxib-dextran ester (GICD) (I0, dextran-glutamic acid-celecoxib).

Abbreviations: TEA, triethylamine; CDI, I,I'-carbonyldiimidazole; ACN, acetonitrile; DMSO, dimethylsulfoxide; DMF, Dimethylformamide; TFA, trifluoroacetic acid. 


\section{Preparation of GIC}

5-Benzyl $N$-(Boc)-glutamic acid (4, $1.336 \mathrm{~g}, 3.96 \mathrm{mmol})$ was dissolved in $15 \mathrm{~mL}$ of ACN followed by the addition of CDI (0.76 g, $4.69 \mathrm{mmol})$, which was stirred at room temperature for 10 minutes. The mixture was added to celecoxib (1, $0.400 \mathrm{~g}, 1.05 \mathrm{mmol})$ and dissolved in ACN containing TEA $(3.44 \mathrm{~mL})$ and reacted at $55^{\circ} \mathrm{C}$ for 4 hours. After removal of solvents by evaporation, the residue was dissolved in ethylacetate/ether $(1 / 1,60 \mathrm{~mL})$, washed with $5 \% \mathrm{NaHCO}_{3}$, dried over anhydrous $\mathrm{Na}_{2} \mathrm{SO}_{4}$, and subjected to flash evaporation to obtain an oily residue. The oily residue was treated with $1 \mathrm{M} \mathrm{NaOH}(15 \mathrm{~mL})$ at room temperature for 3 hours and subsequently with $1 \mathrm{M} \mathrm{HCl}(16 \mathrm{~mL})$ at room temperature for 2 hours to obtain $N$-(Boc)-glutam-1-ylcelecoxib (5) as white precipitate. The precipitate was placed in small volume of $1 \mathrm{M} \mathrm{HCl}$ acetic acid for approximately 1 hour to remove the protection group (6), which was flash-evaporated to obtain G1C (7). Synthetic scheme is shown in Figure 1B. mp: $110^{\circ} \mathrm{C}-115^{\circ} \mathrm{C}$; IR (Nujol, $\left.\mathrm{cm}^{-1}\right): 1,719\left(\mathrm{C}=\mathrm{O}, \mathrm{SO}_{2} \mathrm{~N}-\right.$ $\mathrm{HCO}), 1,595$ (C=O, zwitterionic carboxylate salt); ${ }^{1} \mathrm{H}-\mathrm{NMR}$ (DMSO- $\left.d_{6}, \mathrm{ppm}\right): 1.7(\mathrm{~m}, 1 \mathrm{H}), 2.25(\mathrm{~m}, 1 \mathrm{H}), 2.02(\mathrm{~m}, 2 \mathrm{H})$, $2.30(\mathrm{~s}, 3 \mathrm{H}), 3.29(\mathrm{~m}, 1 \mathrm{H}), 7.14(\mathrm{~s}, 1 \mathrm{H}), 7.16-7.21(\mathrm{~m}, 4 \mathrm{H})$, 7.59 (d, 2H, $J=8.5 \mathrm{~Hz}), 7.95(\mathrm{~d}, 2 \mathrm{H}, J=8.5 \mathrm{~Hz})$; Elemental analysis: $\mathrm{C}_{22} \mathrm{H}_{21} \mathrm{~F}_{3} \mathrm{~N}_{4} \mathrm{O}_{5} \mathrm{~S}(510.12)$ Calcd: $\mathrm{C}, 51.76 ; \mathrm{H}, 4.15$; N, 10.98; Found: C, 51.82; H, 4.11; N, 11.20.

\section{Preparation of glutam- I-ylcelecoxib- dextran ester (dextran-glutamic acid- celecoxib conjugate)}

$\mathrm{CDI}$ (84.59 mg, $0.52 \mathrm{mmol}$ ) was added to $N$-(Boc)-glutam-1-yl celecoxib (8,300 mg, $0.492 \mathrm{mmol})$ dissolved in dimethylformamide $(280 \mu \mathrm{L})$, which was stirred at room temperature for 1 hour. The reaction mixture (9) was treated with dextran $(250 \mathrm{mg})$ dissolved in DMSO $(500 \mu \mathrm{L})$ and TEA $(60.9 \mu \mathrm{L}$, $0.437 \mathrm{mmol}$ ), and reacted at $55^{\circ} \mathrm{C}$ for 4 hours. Excess ethanol was added to the mixture resulting in the formation of white precipitate, which was isolated by centrifugation. The isolated precipitate was treated with trifluoroacetic acid $(0.2 \mathrm{~mL})$ at room temperature for 40 minutes. Excess ethanol was added to precipitate the final product glutam-1-yl celecoxibdextran ester [G1CD] (10, dextran-glutamic acid-celecoxib conjugate) as white powder. Synthetic scheme is shown in Figure 1C. Degree of substitution (DS) was defined as milligrams of celecoxib in $100 \mathrm{mg} \mathrm{G1CD}$ and was measured by HPLC analysis of G1C after hydrolysis of G1CD in $1 \mathrm{M}$ $\mathrm{NaOH}$ for 1 hour. In this study, G1CD (DS: 9.5-10.5) was used for experiments.

\section{Chemical stability}

Each celecoxib derivative was incubated in $\mathrm{pH} 1.2 \mathrm{HCl}$ buffer or $\mathrm{pH} 6.8$ phosphate buffer at $37^{\circ} \mathrm{C}$ for 10 hours. At a predetermined time interval, a $20 \mu \mathrm{L}$ portion of each solution was removed and the concentrations of celecoxib and G1C were analyzed by HPLC as described.

\section{DNS method}

DNS reagent solution was prepared by dissolving DNS ( $5 \mathrm{~g}$ ) in $2 \mathrm{M} \mathrm{NaOH}(100 \mathrm{~mL})$ and distilled water $(250 \mathrm{~mL})$. To this solution, sodium potassium tartratetetrahydrate $(150 \mathrm{~g})$ was added and dissolved and the volume was adjusted to $500 \mathrm{~mL}$ with distilled water. Maltose dissolved in $0.1 \mathrm{M}$ acetate buffer (pH 5.4) at the concentration range of $0.093-0.75 \mathrm{mg} / \mathrm{mL}$ $(200 \mu \mathrm{L})$ was mixed with DNS reagent solution $(600 \mu \mathrm{L})$, boiled for 5 minutes, cooled for 10 minutes, and the absorbance measured at $540 \mathrm{~nm}$ by a UV spectrophotometer (Shimadzu, Tokyo, Japan). A calibration curve was generated using the results. G1CD (DS: 10, equivalent to $2.52 \mathrm{mg}$ dextran $/ 1 \mathrm{~mL}$ ) dissolved in $0.1 \mathrm{M} \mathrm{pH} 5.4$ acetate buffer was incubated with dextranase $(15 \mathrm{DU} / \mathrm{mL})$ at $37^{\circ} \mathrm{C}$. At an appropriate time interval, a portion of the incubated sample was treated with DNS reagent solution according to the same procedure, and the amount of terminal reducing sugar was deduced from the calibration curve. Dextran $(2.52 \mathrm{mg})$ was incubated with dextranase $(15 \mathrm{DU} / \mathrm{mL})$ at $37^{\circ} \mathrm{C}$ for 1.5 hours and this sample was used to calculate the percent degradation of G1CD.

\section{Incubation of celecoxib derivatives in suspensions of gastrointestinal tract contents}

Male Sprague Dawley rats (250-255 g) were sacrificed by $\mathrm{CO}_{2}$ and a midline incision was made. The contents of the small intestine and the cecal contents were separately collected and were suspended in isotonic phosphate buffer ( $\mathrm{pH}$ 6.8). In each microtube, each celecoxib derivative in buffer $(0.5 \mathrm{~mL}$, $1 \mathrm{mM})$ was added to the suspension $(0.5 \mathrm{~mL})$ and incubated at $37^{\circ} \mathrm{C}$ under nitrogen (for incubation in the cecal contents). G1CD dissolved in the buffer (corresponding to $1 \mathrm{mM} \mathrm{G1C}$ ) was used for this experiment. At an appropriate time interval, the samples were extracted with ethylacetate $(0.5 \mathrm{~mL})$ followed by centrifugation at $5,000 \times \mathrm{g}$ for 5 minutes. Methanol $(1.0 \mathrm{~mL})$ was added to the residue obtained from evaporation of the organic layer $(0.1 \mathrm{~mL})$, vortexed, and centrifuged at $10,000 \times g$ for 10 minutes. The concentration of celecoxib in 
a $20 \mu \mathrm{L}$ portion of the supernatant was determined by HPLC. $\mathrm{SC}$ and $\mathrm{G1C}$ were analyzed in samples without extraction.

\section{Oral administration of GICD and preparation of samples for blood and contents of rat intestinal tract}

Male Sprague Dawley rats (250-255 g) were maintained on a stock diet and water ad libitum. These animals were fasted overnight (16 hours) prior to and during the experiments, while allowed free access to water. Water bottles were removed from the cages at least 30 minutes before drug administration. Celecoxib suspension $(2.5 \mathrm{mg} / 0.3 \mathrm{~mL} / \mathrm{rat})$ in $0.5 \%$ sodium carboxymethylcellulose or G1CD solution (equivalent to $2.5 \mathrm{mg}$ celecoxib/1 mL/rat) in distilled water was administered orally to rats by gavage.

At an appropriate time interval, the rats were anesthetized with isoflurane and $1 \mathrm{~mL}$ of blood was collected by intracardiac puncture through a heparinized syringe. Heparinized blood samples were immediately centrifuged at $6,000 \times g$ for 5 minutes, and the plasma was separated and transferred to microcentrifuge tubes. After blood collection, the rats were sacrificed using $\mathrm{CO}_{2}$ gas asphyxiation and the intestinal tract (proximal small intestine, distal small intestine, and cecum) were obtained by midline incision from the rat. The contents in the cecum was separated, transferred to falcon tubes, and mixed with $\mathrm{pH} 6.8$ isotonic phosphate buffer to afford $20 \%(\mathrm{w} / \mathrm{v})$ suspensions. Cecal samples were extracted with an equivalent volume of ethyl acetate for 5 minutes. For extraction of blood samples, fivefold volume of ethyl acetate was used. Each sample was centrifuged at $6,000 \times g$ for 5 minutes for effective phase separation. For analysis of celecoxib, a $1 \mathrm{~mL}$ (for cecal and small intestinal contents) or $0.1 \mathrm{~mL}$ volume (for blood) of $\mathrm{pH} 6.8$ isotonic phosphate buffer was added to the residues obtained from evaporation of an aliquot of the organic layer $(1 \mathrm{~mL})$ in the microcentrifuge tubes, vortexed, and centrifuged at 10,000 $\times g$ at $4^{\circ} \mathrm{C}$ for 10 minutes. The concentration of celecoxib in a $20 \mu \mathrm{L}$ portion of the supernatant was determined by HPLC.

\section{6-Keto-PGF ${ }_{1 \alpha}$ immunoassay}

The serum level of 6-keto-PGF ${ }_{1 \alpha}$ was assessed using a commercially available immunoassay kit (enzyme-linked immunosorbent assay) according to the manufacturer's instructions. Blood (1 mL) was drawn by intracardiac puncture. The clotted blood samples were centrifuged at $2,000 \times \mathrm{g}$ for 30 minutes in the presence of indomethacin $(10 \mu \mathrm{g} / \mathrm{mL})$. The supernatants were collected and stored at $-70^{\circ} \mathrm{C}$ until the 6-keto-PGF ${ }_{1 \alpha}$ assay was performed.

\section{Statistical analysis}

The results are expressed as mean \pm SEM. One-way ANOVA followed by Tukey's (honest significant difference [HSD]) test was used for testing the difference between data. Differences with $P<0.05$ were considered significant. XLSTAT ${ }^{\circledR}$ Software (Addinsoft, Inc, Suite 503; New York, NY, USA) was used for statistical analysis.

\section{Results \\ GIC but not SC releases celecoxib in cecal contents}

After colonic delivery of a dextran-glutamic acid-celecoxib conjugate, the celecoxib-linker is released from oligomerized dextran-linker-celecoxib and needs to be cleaved in order to release celecoxib. ${ }^{15}$ To compare colonic celecoxib release from $\mathrm{G} 1 \mathrm{C}$ with that from SC, ${ }^{19} \mathrm{G} 1 \mathrm{C}$ and SC were incubated with the cecal contents of rats and the release of celecoxib was monitored. While SC released up to approximately $5 \%$ of the initial dose at 24 hours, G1C released up to approximately $55 \%$ and $75 \%$ of the initial dose at 10 and 24 hours, respectively (Figure 2). These results indicate that glutamic acid is a more useful linker than succinic acid.

\section{GICD releases celecoxib in cecal contents but not in small intestinal contents}

Based on the earlier in vitro data, a dextran-glutamic acid-celecoxib conjugate, which has a glutamic acid linker

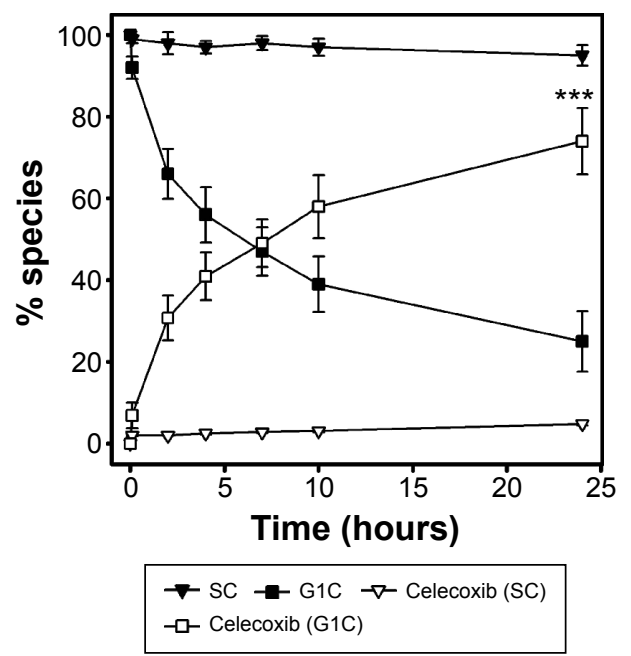

Figure 2 Release of celecoxib during incubation of succinyl celecoxib (SC) or glutam-I-yl celecoxib (GIC) with the cecal contents of rats.

Notes: SC or GIC was incubated with $10 \%$ cecal contents at $37^{\circ} \mathrm{C}$ for 24 hours. Drugs in the cecal suspension were analyzed by HPLC at the indicated time intervals. The data represent mean \pm SEM $(n=3)$. $* * * P<0.001$ vs celecoxib from SC.

Abbreviations: HPLC, high-performance liquid chromatography; SEM, standard error of mean. 
between dextran and celecoxib, was prepared. To examine whether G1CD could be colon specific and deliver celecoxib to the large intestine without significant loss in the upper intestine, the polymeric conjugate was incubated for 6 hours in $\mathrm{pH} 1.2$ or 6.8 buffer solutions, representing $\mathrm{pH}$ of the stomach and small intestine, respectively. In addition, G1CD was incubated with small intestine contents for 10 hours. Either celecoxib or G1C was not detected in the experiments.

To examine whether G1CD delivered to the large intestine would liberate celecoxib, the conjugate was incubated with the cecal contents of rats and celecoxib release was monitored. As shown in Figure 3A, up to approximately $18 \%$ and $53 \%$ celecoxib were released at 8 and 24 hours, respectively. To test whether celecoxib release was dependent on microbial enzymes in the large intestine, the same experiment was performed with autoclaved cecal contents, which are used to test microbially triggered activation of a colonspecific prodrug. ${ }^{21}$ In contrast, celecoxib was not detected until 12 hours after incubation of G1CD with the autoclaved cecal contents.

\section{GICD is a substrate of dextranase}

The earlier data suggest that although esterases are abundant throughout the digestive tract, the ester bond between celecoxib-glutamic acid and dextran is cleaved (deesterified) only in the large intestine where microbial dextranases exist; thus the dextran backbone of G1CD can be degraded to produce oligomers, which reduce the steric hindrance imposed by dextran, most likely facilitating the cleavage of the ester link between dextran and G1C. ${ }^{15}$ This rationale prompted us to examine whether the modified dextran,
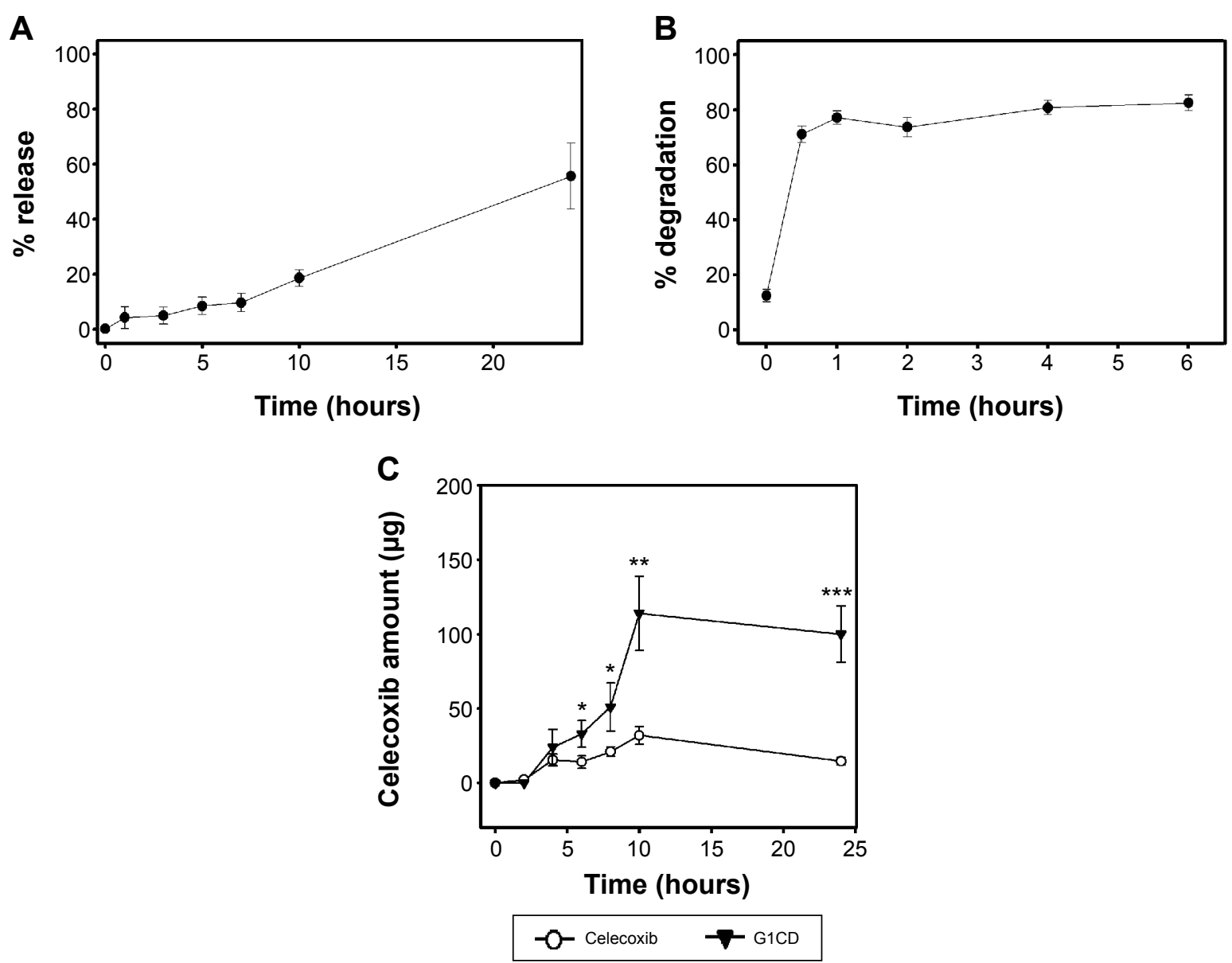

Figure 3 Glutam-I-yl celecoxib-dextran ester (GICD) administered orally delivers and liberates celecoxib at the large intestine.

Notes: (A) GICD was incubated with the cecal contents at $37^{\circ} \mathrm{C}$ for 24 hours. Celecoxib in the cecal suspension was analyzed by HPLC at the indicated time intervals. The data represent mean \pm SEM ( $n=3)$; (B) the extent of depolymerization was measured using the DNS method at predetermined time intervals during incubation of GICD (DS: 10) with dextranase (I5 DU/mL). The same experiment was performed with dextran. The percent degradation of GICD represents the ratio of the depolymerization of GICD to that of dextran treated with dextranase for I.5 hours. The data represent mean \pm SEM $(n=3)$; (C) celecoxib in the cecum was analyzed by HPLC at appropriate time intervals after oral administration of GICD or celecoxib. The data represent mean \pm SEM $(n=5)$. $* P<0.05$, $* * P<0.005$, $* * * P<0.00$ I vs cecal celecoxib after oral administration of celecoxib at each time point.

Abbreviations: HPLC, high-performance liquid chromatography; DNS, dinitrosalicylic acid; DS, degree of substitution; SEM, standard error of mean. 
G1CD, was depolymerized by dextranase. Depolymerization was assessed using the DNS method following the incubation of G1CD with dextranase. Depolymerization appeared to be completed in 1 hour (Figure 3B).

\section{GICD delivers celecoxib to the large intestine}

To examine the specificity of G1CD for the colon in vivo, G1CD was administered orally to rats and the amount of celecoxib in the contents of the small intestine and the cecum were measured at appropriate time intervals. For comparison, the same experiment was performed with free celecoxib. In the small intestine, celecoxib was detected at $52.4 \pm 12.4 \mu \mathrm{g}$ in proximal small intestine (PSI) and $129 \pm 29.2 \mu \mathrm{g}$ in DSI 4 hours after gavage of free celecoxib. In contrast, either celecoxib or G1C was not detected in the proximal and distal small intestine upon oral administration of G1CD. On the other hand, celecoxib began to appear in the cecum 4 hours after oral administration of G1CD. The peak concentrations of celecoxib were reached at 10 hours. The recovery of celecoxib was better after G1CD administration than that after celecoxib administration (Figure $3 \mathrm{C}$ ).

\section{GICD does not affect the serum level of 6-keto-PGF $1 \alpha$}

Long-term use of celecoxib for the prevention of CRC or the treatment of colitis may cause cardiovascular toxicity, which is correlated with the concentration of celecoxib in blood. ${ }^{11}$ To examine whether G1CD could reduce the blood concentration of celecoxib, celecoxib or G1CD was administered orally to rats and the plasma concentrations of celecoxib were compared. As shown in Figure 4A, while the plasma concentration of celecoxib reached $2.8 \mu \mathrm{M}$ at 2 hours after oral administration of celecoxib, celecoxib was not detectable in the plasma for 24 hours after oral administration of G1CD, indicating that G1CD limited the systemic absorption of celecoxib.

We next examined whether limiting the systemic absorption of celecoxib from G1CD could reduce the risk of cardiovascular toxicity of celecoxib. Celecoxib or G1CD was administered orally to rats and the serum levels of 6-keto-PGF ${ }_{1 \alpha}$, an inverse indicator of cardiovascular toxicity of celecoxib, ${ }^{22}$ were monitored. As shown in Figure 4B, administration of free celecoxib decreased 6-keto-PGF levels by up to $40 \%$ of the initial level 4 hours later, and the level returned to the baseline level at approximately 10 hours later; on the other hand, G1CD did not affect the serum levels of 6-keto-PGF ${ }_{1 \alpha}$ throughout the experimental period. These results were in line with the blood concentration data.

\section{Discussion}

In this study, G1CD was depolymerized and released celecoxib in the large intestine while remaining stable in the upper intestine. Oral administration of G1CD delivered a
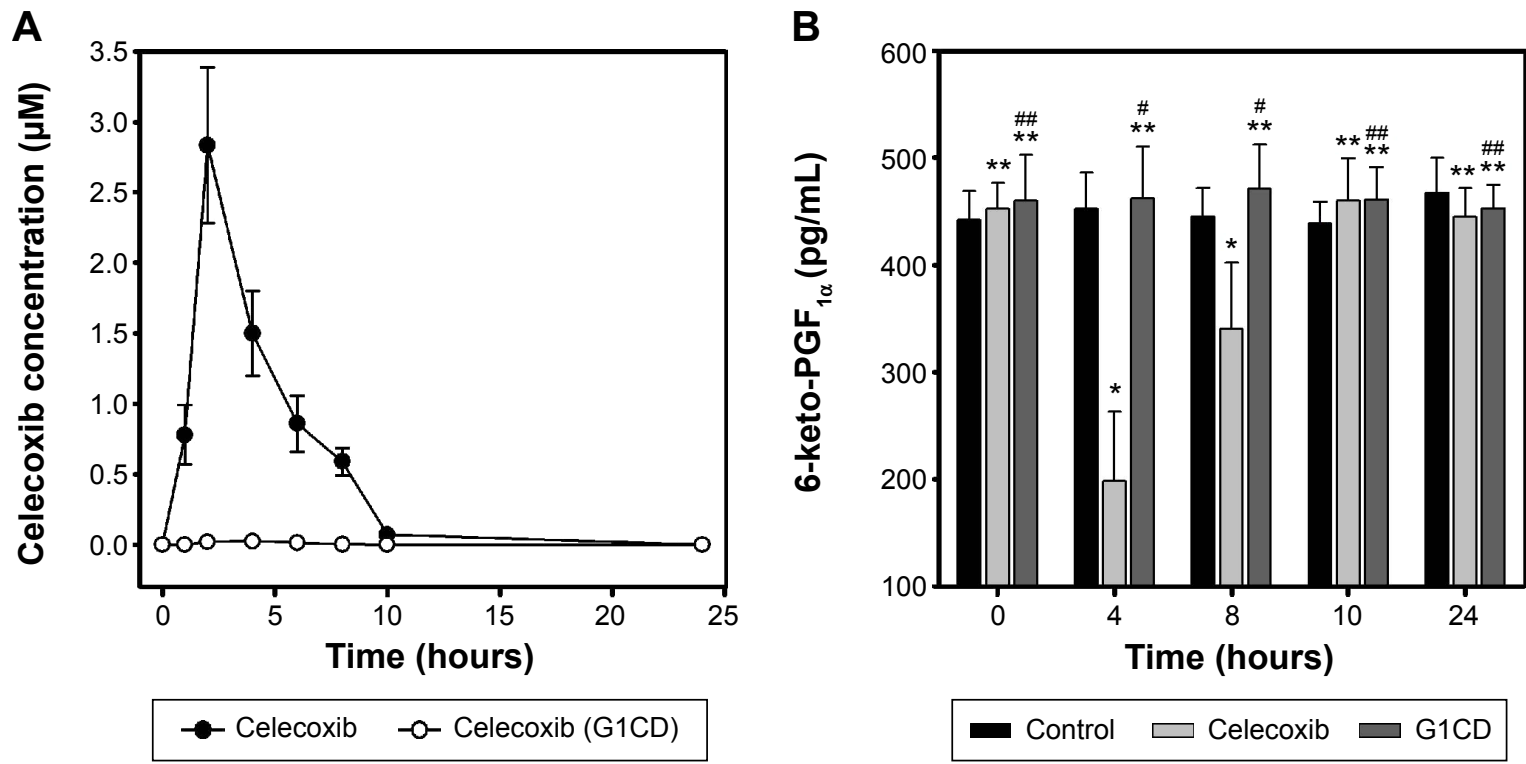

Figure 4 Glutam-I-yl celecoxib-dextran ester (GICD) does not affect the serum level of 6-ketoprostaglandin $F_{1 \alpha}\left(6-k e t o-P G F_{\mid \alpha}\right)$.

Notes: (A) Celecoxib in the blood was analyzed by HPLC at appropriate time intervals after oral administration of GICD or celecoxib. The data represent mean \pm SEM $(n=5)$. (B) 6-keto-PGF $1 \alpha$ in the blood was analyzed using an ELISA kit at appropriate time intervals after oral administration of GICD or celecoxib. The data represent the mean \pm SEM ( $n=5)$. $* P<0.05$ vs control; ${ }^{* * P}>0.05$ vs control; ${ }^{*} P<0.05$ vs celecoxib; ${ }^{\#} P>0.05$ vs celecoxib at each time point.

Abbreviations: HPLC, high-performance liquid chromatography; ELISA, enzyme-linked immunosorbent assay. 
greater amount of celecoxib to the large intestine than that delivered using free celecoxib. Moreover, G1CD prevented the systemic absorption of celecoxib and did not decrease the serum level of 6-keto-PGF ${ }_{1 \alpha}$, an inverse indicator of cardiovascular side effects of celecoxib.

Consistent with previous papers, G1CD, a dextran-based colon-specific prodrug, shows specificity for the colon. Our in vitro data show that, while G1CD did not release celecoxib in simulated stomach and small intestine conditions, a significant release of celecoxib was observed in cecal contents. In parallel with this observation, oral administration of G1CD delivered greater amount of celecoxib to the large intestine without premature release of celecoxib in the small intestine. The colon specificity of G1CD should lead to a greater concentration at the target site and be therapeutically advantageous for the treatment of colonic diseases. In fact, a colon-specific celecoxib prodrug using a small-molecule carrier is reported to have enhanced anti-colitic effects in a trinitrobenzene sulfonic acid (TNBS)-induced rat colitis model. ${ }^{8}$ The authors suggest that the enhanced anti-colitic effects are attributable to the increased colonic celecoxib concentration, which exerts these effects via pharmacologic mechanisms that may not be available when free celecoxib is used. ${ }^{8}$

Despite greater accumulation of celecoxib at the target site (large intestine) probably leading to therapeutic enhancement, G1CD would prevent the systemic absorption of celecoxib, thus attenuating the cardiovascular side effects of celecoxib. Our data show that celecoxib was not detectable in the blood after oral administration of G1CD and in agreement with this observation, G1CD did not decrease the serum level of 6-keto-PGF ${ }_{1 \alpha}$ associated with cardiovascular risk of celecoxib. ${ }^{22}$ In contrast, oral administration of free celecoxib afforded a celecoxib concentration of up to $2.8 \mu \mathrm{M}$ in blood and substantially decreased the prostaglandin levels.

In this study, we focused on whether G1CD can elicit colon targetability and its toxicological and therapeutic advantages over free celecoxib. Considering that polymerdrug conjugates generally exhibit sustained release of the drug, ${ }^{23}$ G1CD could also control the release of celecoxib in the large intestine, thus resulting in desirable colonic celecoxib distribution. Since pathological lesions for CRC and inflammatory bowel disease exist in the distal region of the large intestine, ${ }^{24}$ it would be beneficial to control colonic drug distribution even with colon-specific drugs. However, a more elaborate distribution study would be needed to identify whether G1CD offers such a benefit. Collectively, G1CD may be a polymeric colon-specific celecoxib prodrug with toxicological and therapeutic advantages.

\section{Acknowledgment}

This work was supported by the National Research Foundation of Korea (NRF) grant funded by the Korean government (MSIP) (No 2009-0083538).

\section{Disclosure}

The authors report no conflicts of interest in this work.

\section{References}

1. Herendeen JM, Lindley C. Use of NSAIDs for the chemoprevention of colorectal cancer. Ann Pharmacother. 2003;37(11):1664-1674.

2. Hilmi I, Goh KL. Chemoprevention of colorectal cancer with nonsteroidal anti-inflammatory drugs. Chin J Dig Dis. 2006;7(1):1-6.

3. Bus PJ, Verspaget HW, Lamers CB, Griffioen G. Chemoprevention of colorectal cancer by non-steroidal anti-inflammatory drugs. Scand J Gastroenterol Suppl. 2000;232:101-104.

4. Fort J. Celecoxib, a COX-2 - specific inhibitor: the clinical data. Am J Orthop. 1999;28(3 suppl):13-18.

5. Arber N, Eagle CJ, Spicak J, et al; PreSAP Trial Investigators. Celecoxib for the prevention of colorectal adenomatous polyps. $N$ Engl J Med. 2006;355(9):885-895.

6. Davies NM, Gudde TW, de Leeuw MA. Celecoxib: a new option in the treatment of arthropathies and familial adenomatous polyposis. Expert Opin Pharmacother. 2001;2(1):139-152.

7. Steinbach G, Lynch PM, Phillips RK, et al. The effect of celecoxib, a cyclooxygenase- 2 inhibitor, in familial adenomatous polyposis. $N$ Engl J Med. 2000;342(26):1946-1952.

8. Lee Y, Kim W, Hong S, et al. Colon-targeted celecoxib ameliorates TNBS-induced rat colitis: a potential pharmacologic mechanism and therapeutic advantages. Eur J Pharmacol. 2014;726: $49-56$.

9. Cuzzocrea S, Mazzon E, Serraino I, et al. Celecoxib, a selective cyclooxygenase- 2 inhibitor reduces the severity of experimental colitis induced by dinitrobenzene sulfonic acid in rats. Eur J Pharmacol. 2001; 431(1):91-102.

10. El-Medany A, Mahgoub A, Mustafa A, Arafa M, Morsi M. The effects of selective cyclooxygenase- 2 inhibitors, celecoxib and rofecoxib, on experimental colitis induced by acetic acid in rats. Eur J Pharmacol. 2005;507(1-3):291-299.

11. Brophy JM. Cardiovascular risk associated with celecoxib. $N$ Engl $J$ Med. 2005;352(25):2648-2650. [author reply 2648-2650].

12. Caldwell B, Aldington S, Weatherall M, Shirtcliffe P, Beasley R. Risk of cardiovascular events and celecoxib: a systematic review and metaanalysis. J R Soc Med. 2006;99(3):132-140.

13. Oviedo JA, Schroy PC 3rd. Does celecoxib use increase the risk of cardiovascular events? Gastroenterology. 2005;129(4):1348-1350.

14. Solomon SD, McMurray JJ, Pfeffer MA, et al; Adenoma Prevention with Celecoxib (APC) Study Investigators. Cardiovascular risk associated with celecoxib in a clinical trial for colorectal adenoma prevention. N Engl J Med. 2005;352(11):1071-1080.

15. Jung Y, Kim YM. What should be considered on design of a colonspecific prodrug? Expert Opin Drug Deliv. 2010;7(2):245-258.

16. Rubinstein A. Microbially controlled drug delivery to the colon. Biopharm Drug Dispos. 1990;11(6):465-475.

17. Sinha VR, Kumria R. Colonic drug delivery: prodrug approach. Pharm Res. 2001;18(5):557-564

18. Sinha VR, Kumria R. Polysaccharides in colon-specific drug delivery. Int J Pharm. 2001;224(1-2):19-38. 
19. Shrivastava PK, Shrivastava SK. Dextran carrier macromolecule for colon specific delivery of celecoxib. Curr Drug Deliv. 2010;7(2): 144-151.

20. Lee Y, Kim J, Kim H, et al. N-succinylaspart-1-yl celecoxib is a potential colon-specific prodrug of celecoxib with improved therapeutic properties. J Pharm Sci. 2012;101(5):1831-1842.

21. Kim H, Kong H, Choi B, et al. Metabolic and pharmacological properties of rutin, a dietary quercetin glycoside, for treatment of inflammatory bowel disease. Pharm Res. 2005;22(9):1499-1509.
22. Buerkle MA, Lehrer S, Sohn HY, Conzen P, Pohl U, Krotz F. Selective inhibition of cyclooxygenase-2 enhances platelet adhesion in hamster arterioles in vivo. Circulation. 2004;110(14):2053-2059.

23. Mehvar R. Dextrans for targeted and sustained delivery of therapeutic and imaging agents. J Control Release. 2000;69(1):1-25.

24. Friend DR. Review article: issues in oral administration of locally acting glucocorticosteroids for treatment of inflammatory bowel disease. Aliment Pharmacol Ther. 1998;12(7):591-603.

\section{Publish your work in this journal}

Drug Design, Development and Therapy is an international, peerreviewed open-access journal that spans the spectrum of drug design and development through to clinical applications. Clinical outcomes, patient safety, and programs for the development and effective, safe, and sustained use of medicines are a feature of the journal, which has also been accepted for indexing on PubMed Central. The manuscript management system is completely online and includes a very quick and fair peer-review system, which is all easy to use. Visit http://www.dovepress.com/testimonials.php to read real quotes from published authors.

\footnotetext{
Submit your manuscript here: http://www.dovepress.com/drug-design-development-and-therapy-journal
} 